\title{
3D transesophageal echocardiography assists in evaluating the morphology, function, and presence of thrombi of left atrial appendage in patients with atrial fibrillation
}

\author{
Bingqing Deng, Ruqiong Nie, Qiong Qiu, Yulin Wei, Yingmei Liu, Hanlu Lv, Shaoxin Zheng, \\ Jingfeng Wang \\ Cardiovascular Department, Sun Yat-sen Memorial Hospital, Sun Yat-sen University, Guangzhou, China \\ Contributions: (I) Conception and design: B Deng, R Nie; (II) Administrative support: J Wang, R Nie; (III) Provision of study materials or patients: J \\ Wang, R Nie; (IV) Collection and assembly of data: B Deng, Q Qiu, Y Wei, Y Liu, H Lv, S Zheng; (V) Data analysis and interpretation: B Deng; (VI) \\ Manuscript writing: All authors; (VII) Final approval of manuscript: All authors. \\ Correspondence to: Jingfeng Wang. Cardiovascular Department, Sun Yat-sen Memorial Hospital, Sun Yat-sen University, Guangzhou, China. \\ Email: wjingf@mail.sysu.edu.cn.
}

Background: Left atrial appendage (LAA) is significantly more likely to form thrombi in patients with atrial fibrillation (AFib). Two-dimensional transesophageal echocardiography (2D TEE) is considered the gold standard for assessing and studying LAA morphology and anatomy. However, 2D TEE can only visualize one plane at any given time. Real-time three-dimensional echocardiography (RT-3D TEE) imaging can preserve spatial and temporal resolution, which is a safe, accurate, and reproducible imaging modality. There are few reports of the usage of RT-3D TEE to study LAA in AFib patients. In our research, RT-3D TEE helps to provide detailed LAA information and identifying the presence or absence of thrombi from pectinate muscles in paroxysmal and long-standing AFib patients.

Methods: LAA morphology was analyzed in detail by 2D TEE and RT-3D TEE in 320 patients with paroxysmal or long-standing AFib. The LAA flow pattern, as maximal LAA emptying flow velocity (LAAeV), was retrieved from 2D and 3D TEE imaging. LAA morphological parameters, spontaneous echo contrast (SEC), and thrombi were also detected by 2D and 3D TEE in all patients. In addition, LAA lobes and types were classified according to the morphology by $3 \mathrm{D}$ TEE, and the relationship between LAA types and the incidence of thrombi was evaluated.

Results: Long-standing AFib had greater enlargement of LAAs (orifice diameters and area), significantly more severe SEC, and a higher thrombi clot incidence rate by 3D-TEE compared with paroxysmal AFib patients $(\mathrm{P}<0.05)$. In addition, cauliflower morphology in long-standing AFib patients was associated with a higher LAA thrombus (OR 2.1, 95\% CI: 1.1-8.5, P=0.031) and increased prevalence of SEC. Moreover, the uncertainty of thrombi detection was significantly decreased by 3D TEE compared with 2D TEE $(\mathrm{P}<0.001)$, and the certainty of thrombi detection by $3 \mathrm{D}$ TEE also decreased slightly $(\mathrm{P}=0.06)$.

Conclusions: RT-3D TEE is a safe and real-time option for the evaluation of LAA morphology and function. Long-standing AFib has greater LAA and SEC, as well as a higher incidence of thrombi than the paroxysmal group. Cauliflower LAA type was associated with a higher prevalence of SEC and thrombi.

Keywords: Atrial fibrillation (AFib); real-time 3D trans-esophageal echocardiography (RT-3D TEE); left atrial appendage (LAA)

Submitted Mar 29, 2021. Accepted for publication May 21, 2021.

doi: $10.21037 / \mathrm{atm}-21-1981$

View this article at: http://dx.doi.org/10.21037/atm-21-1981 


\section{Introduction}

Due to its complex, variable morphology and abundant pectinate muscles, left atrial appendage (LAA) is significantly more likely to form thrombi in patients with non-valve atrial fibrillation (AFib). Generally, more than $30 \%$ of thromboembolic events are cardiogenic, with $90 \%$ of these in non-valve AFib originating from LAA which become misshape and have swirl blood pattern and slow LAA empty velocity. Understanding the morphology and function of LAA is pivotal for the treatment of AFib. However, in most cases, it is impossible to clearly detect LAA by ordinary two-dimensional transthoracic echocardiography (2D TTE) alone, due to its small size and distant position from the transducer, as well as the patients' body habitus, etc. Two-dimensional transesophageal echocardiography (2D TEE) is considered the gold standard for assessing and studying LAA morphology and anatomy, owing to its higher sensitivity and specificity. In addition, 2D TEE can focus on LAA cardiac hemodynamics, such as the LAA orifice flow pattern, peak velocity, state of sludge, spontaneous echocardiographic contrast (SEC), and even thrombus formation. However, 2D TEE can only visualize one plane at any given time, which will prevent comprehensive and deep scanning of LAA. Therefore, relying only on 2D TEE will lead to missed details or even wrong conclusions with regards to thrombus clot diagnosis from pectinate muscles.

Real-time three-dimensional echocardiography (RT-3D TEE) imaging can preserve spatial and temporal resolution, and thereby significantly enhance the visualization of complex 3D structures, such as the LAA. It is a safe, accurate, and reproducible imaging modality that is used as a complementary and supplementary method to $2 \mathrm{D}$ imaging to assess heart function and anatomy in different heart diseases. Available evidence suggests that $3 \mathrm{D}$ echocardiography provides much improved imaging quality over 2D methods for left ventricular volume and function calculation, as well as heart valve evaluation, especially in the mitral and aortic valves. RT-3D TEE, which provides multi-beam scanning in any cardiac plane, combined with TEE technology, will offer improved echo image quality and visualization of the cardiac structure.

There are few reports of the usage of RT-3D TEE to study the morphology and function of LAA in AFib patients. Dentamaro et al. (1) evaluated LAA function and thrombi with 3D TEE, however their study did not provide details about different classifications of AFib patients or a detailed structural description about LAA. Park et al. (2) compared the morphology and function in two classifications of AFib patients with 3-dimensional multislice helical contrast computerized tomography (3D-CT). In our research, we used RT-3D TEE to assist in providing detailed morphological and functional information about LAA, and to identify the presence or absence of thrombi from pectinate muscles in paroxysmal and long-standing AFib patients.

We present the following article in accordance with the STROBE reporting checklist (available at http://dx.doi. org/10.21037/atm-21-1981).

\section{Methods}

\section{Study population}

In this retrospective cohort study, 351 patients from the Department of Cardiology, Sun Yat-sen Memorial Hospital affiliated to Sun Yat-sen University between 1st November, 2019 and 31th December, 2020 were enrolled. The clinical indication for echocardiography involved two types of AFib: paroxysmal and long-standing. Thirty-one patients were excluded due to poor image quality. Of the remaining 330 patients, 110 had paroxysmal AFib, and 210 had longstanding AFib. All of the included patients were antiarrhythmic drug resistant and required cardioversion or radiofrequency ablation. 2D TTE was initially performed in these AFib patients. 2D TEE and RT-3D TEE were then performed to obtain the LAA-related parameters and confirm the absence of LAA thrombi prior to catheter ablation or cardioversion. Figure 1 shows the enrolment flowchart of this patient cohort. This study was performed in accordance with the Declaration of Helsinki (as revised in 2013), and the study protocol was approved by the Sun Yat-sen Memorial Hospital ethics committee. Written informed consent was obtained from all patients for 3D transesophageal echocardiography.

\section{Echocardiography parameters}

Standard 2D transthoracic echocardiographic examinations were performed using a Siemens SC2000 echocardiography with 4V1C probe (Siemens Medical Systems, Illinois, USA) and Philips EPIQ Elite echocardiography with X5-1 probe (Philips Healthcare, Washington, USA). Endsystolic and end-diastolic left atrial volumes were measured using a modified Simpson's method both in apical 4- and 2-chamber views. The left ventricular ejection fraction 

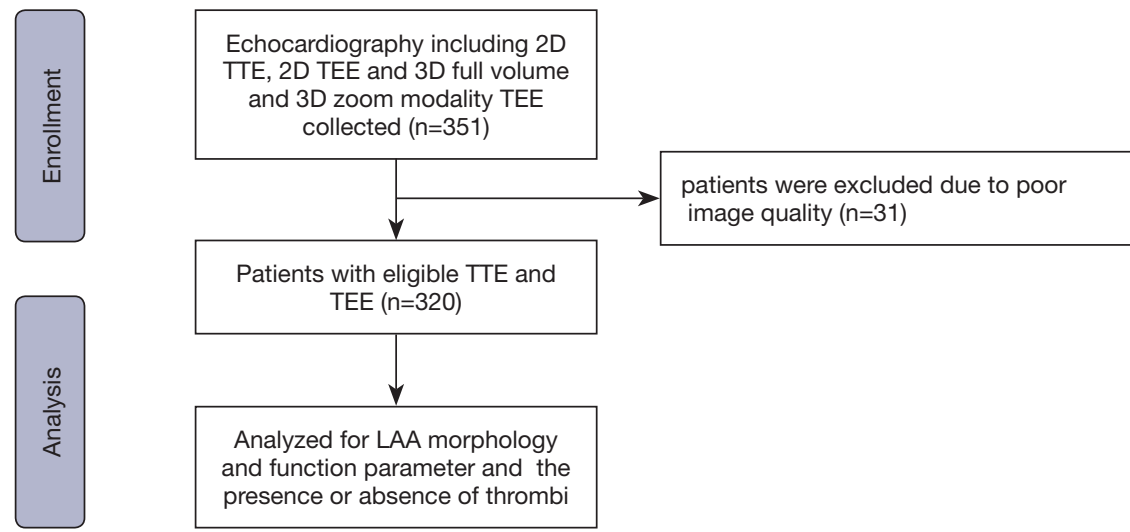

Figure 1 Research flowchart: overview of the enrolment process and analysis of this study cohort. Of 351 examinations screened as eligible for 2D TTE/2D TEE/RT-3D TEE, a total of 320 datasets were found to be of acceptable quality (sufficient image quality =91\%). 2D TTE/2D TEE/RT-3D TEE, two-dimensional transthoracic echocardiography/two-dimensional transesophageal echocardiography/realtime three-dimensional transesophageal echocardiography; LAA, left atrial appendage.

(LVEF) was measured using a modified Simpson's method. The conventional diastolic transthoracic echocardiographic parameters were examined, including trans-mitral inflow Doppler patterns and tissue inflow Doppler patterns in the 4-chamber apical view (E wave, e' septal wave, e' lateral wave and E/e' ratio).

Transesophageal echocardiography images were acquired by Siemens SC2000 echocardiography system with Z6Ms live 3D TEE transducer (Siemens Medical Systems, Illinois, USA) and Philips EPIQ Elite echocardiography with X7-2t live 3D TEE transducer (Philips Healthcare, Washington, USA). Patients were fasted for 12 hours and the TEE procedure was performed in a state of local superficial anesthesia of the pharynx and larynx with lidocaine mucilage. Blood pressure, heart rate, electrocardiogram, and finger vein blood oxygen saturation were closely supervised during the operation. 2D and 3D TEE images were acquired according to the American Society of Echocardiography (ASE) standard protocol (3).

Optimal 2D TEE and Color Doppler TEE images were obtained. Pulse wave Doppler was used to measure maximal emptying flow velocity at the proximal end (4) or orifice of LAA. At last, the average value of $\mathrm{LAAeV}$ in the five cardiac cycles is considered as the representative of LAA function. The LAA volume was also measured using computer software (Philip) and by tracing the endocardium of LAA within the various planes at LAA end-diastole and end-systole. Next, the LAA ejection fraction was measured using the systolic and diastolic volumes of LAA. The LAA depth was measured from the orifice to a lobe tip at the left ventricular end-systole. We measured the LAA orifice diameter at $45^{\circ}$ and $135^{\circ}$ views, and the orifice area at left ventricular end-systole and end-diastole. In the 3D imaging analysis where the $\mathrm{x}$-axis plane and the perpendicular $\mathrm{y}$-axis plane both went through the longitudinal section of the LAA, the image was rotated to display the two planes to view the maximum area of LAA in one of these two planes. The 2D TEE simultaneous $\mathrm{X}$-plane mode, which provides visualized orthogonal images, could assist in the assessment of SEC and thrombi.

The 3D TEE pyramidal full volume images derived from five cardiac beats and the 3D TEE zoom dataset of the entire LAA in each patient were acquired, stored and analyzed online or offline using the Siemens System and Philips Healthcare Q lab software. Each image had a frame rate of 20-30 frames/sec in the full-volume mode, along with a 12 frames/sec in the zoom mode. We defined the end-systole of LAA as the start of the QRS wave and the end-diastole of LAA as the end of the T-wave on the electrocardiogram.

\section{Classification of LAA morphology}

We determined the number and type of LAA lobes from the $135^{\circ} 2 \mathrm{D}$ views and $3 \mathrm{D}$ TEE. LAA morphology was classified into four types: "chicken wing" type, "windsock" type, "cactus" type and "cauliflower" type Wang et al. (5). The presence of an obvious bend or folding back on itself at the proximal or middle part of the dominant lobe was identified as LAA chicken wing type. Non-chicken wing 
LAA morphology was sub-classified as windsock, cactus, and cauliflower (6). The "cactus" shape has a dominant lobe, with secondary lobes arising from the dominant one superiorly and inferiorly. The "cauliflower" type has a short overall length and a variable number of lobes. It also lacks a dominant lobe, and has a more irregularly-shaped orifice. The "windsock" has a dominant lobe as the primary structure, and there are variations in the location and number of lobes.

\section{Criteria for SEC grades}

We included four grades according to the severity SEC by $3 \mathrm{D}$ TEE. The severity of SEC was graded from 0 to 4 based on the density of the swirling blood flow patterns, according to the following criteria (7): 0 (nil): absence of echogenicity; 1 (mild): minimal echogenicity, imperceptible at normal gain settings, may be detectable only transiently during the cardiac cycle, and located in LAA or sparsely distributed in the left atrial cavity; 2 (mild to moderate): more dense swirling pattern, detectable without increased gain settings, with a similar distribution to $1 ; 3$ (moderate): dense swirling pattern in the LAA, generally associated with a relatively lower intensity throughout the main left atria 1 cavity, and may fluctuate in intensity but is detectable constantly throughout the cardiac cycle; and 4 (severe): intense echogenicity and very slow swirling patterns in LAA, usually with similar density in the main cavity.

\section{Statistical analysis}

All statistical analyses were performed using GraphPad version 9.0 (Prism Inc., California, USA). Continuous variables were expressed as mean \pm standard deviation, and categorical variables were presented as counts and percentages. Comparison of parametric continuous data between two groups was performed by the Student's $t$ test. Comparison of categorical values between two groups was performed by chi-square test or Fisher's exact test. A twotailed $\mathrm{P}$ value was calculated for all comparisons, and $\mathrm{P}$ values $<0.05$ were considered statistically significant.

\section{Results}

\section{Study population}

Detailed clinical baseline characteristics are shown in Table 1. Our retrospective study enrolled 330 symptomatic AFib patients who were anti-arrhythmic drugs resistant and required cardioversion or radiofrequency ablation. Among them, 110 patients were paroxysmal AFib, and 220 were long-standing AFib patients. There were 149 (71.0\%) male patients in the long-standing AFib group, and 76 (69.1\%) male patients in the paroxysmal AFib group. In the paroxysmal AFib group, the mean age was $62.0 \pm 10.4$ years, and the body surface area was $1.75 \pm 0.6 \mathrm{~m}^{2}$. The mean age and body surface area in long-standing AFib group was $67.2 \pm 12.8$ years and $1.79 \pm 0.4 \mathrm{~m}^{2}$, respectively. We used CHA2DS2-VASc and HAS-BLED scoring system to assess the risk factors. The long-standing AFib group patients had high grade of thromboembolic (CHA2DS2-VASc $=3.2 \pm 1.8$ ) and hemorrhagic risk (HAS-BLED $=2.6 \pm 1.1$ ), whereas paroxysmal AFib group patients had moderate thromboembolic and hemorrhagic risk $(1.8 \pm 1.2$ and $1.6 \pm 0.9$, respectively). Hypertension, diabetes, ischemia heart disease, congestive heart failure (CHF), and prior stroke/ TIA were present in 70 (63.6\%), 14 (12.7\%), 7 (6.4\%), 16 (14.5\%), and $6(5.4 \%)$ paroxysmal AFib patients, compared with 137 (65.3\%), 28 (13.3\%), 25 (11.9\%), 35 (16.7\%), and $23(11.0 \%)$ long-standing AFib patients. The incidences of ischemia heart disease and stroke were statistically significant between the two groups, whereas there was no statistically significant difference in the incidence of hypertension, T2DM, and heart failure (Table 1).

\section{D TTE and 2D TEE echocardiography results}

The 2D TTE and 2D TEE left atrial and LAA images were collected from all patients. The 2D TTE and 2D TEE features of the enrolled patients are shown in Table 2. The average anteroposterior (AP) diameter of the left atrium in paroxysmal AFib patients by 2D TTE was $35.3 \pm 7.2 \mathrm{~mm}$, compared with $43.0 \pm 6.8 \mathrm{~mm}$ in the long-standing AFib group $(\mathrm{P}<0.001)$. The systolic and diastolic average left atrial mean volume (biplane) were $52.6 \pm 27.3$ and $24.5 \pm 12.0 \mathrm{~mL}$, respectively, in the paroxysmal patients, compared with $72.8 \pm 21.6$ and $48.3 \pm 15.9 \mathrm{~mL}$, respectively, in the longstanding AFib group, and these differences were statistically significant $(\mathrm{P}<0.001)$. In most long-standing AFib patients, the anterior blood flow Doppler modality presented as unimodal. The results were statistically significant in terms of the value of $E$ wave value, e' septal wave, and $E / e^{\prime}$ ratio. However, between the two study groups, there was no difference in terms of the LVEF value $(\mathrm{P}=0.34)$.

We also obtained good quality LAA images by $2 \mathrm{D}$ TEE in all patients, and LAA morphology and functional parameters 
Table 1 Clinical characteristics of patients enrolled in this study

\begin{tabular}{|c|c|c|c|}
\hline Characteristics & Paroxysmal AFib (N=110) & Long persistent AFib $(\mathrm{N}=210)$ & $P$ value \\
\hline Age (years) & $62.0 \pm 10.4$ & $67.2 \pm 12.8$ & 0.03 \\
\hline $\mathrm{BSA}\left(\mathrm{m}^{2}\right)$ & $1.75 \pm 0.6$ & $1.79 \pm 0.4$ & 0.20 \\
\hline \multicolumn{4}{|c|}{ Cardiovascular risk factors, $\mathrm{n}(\%)$} \\
\hline T2DM & $14(12.7)$ & $28(13.3)$ & 0.34 \\
\hline Ischemia heart disease & $7(6.4)$ & $25(11.9)$ & 0.01 \\
\hline Heart failure & $16(14.5)$ & $35(16.7)$ & 0.05 \\
\hline Prior stroke/TIA & $6(5.4)$ & $23(11.0)$ & $<0.001$ \\
\hline HAS-BLED Score & $1.6 \pm 0.9$ & $2.6 \pm 1.1$ & $<0.001$ \\
\hline
\end{tabular}

All data are presented as number (\%) or mean \pm SD. AFib, atrial fibrillation; BSA, body surface area; CHA2DS2-VASc score: congestive heart failure, hypertension, age $\geq 75$ (doubled), diabetes, stroke (doubled), vascular disease, age 65-74, sex category (female); HAS-BLED: hypertension, abnormal renal and/or liver function, stroke, bleeding, labile INR, age $\geq 65$, alcohol use.

were accurately collected. We found that long-standing AFib patients had a persistent dysfunctional LAA with a lower empty velocity (LAAeV) (mean value $25.1 \pm 9.9 \mathrm{~cm} / \mathrm{s}$ ) and lower LAA EF\% (mean value $50.7 \% \pm 10.3 \%$ ) compared with the paroxysmal group, and the differences were statistically significant (LAAeV $\mathrm{P}<0.001$, LAA EF\% $\mathrm{P}=0.01$ ). In addition, 2D TEE could easily observe a SEC in 51 patients (from both groups), whereas using the 2D TTE method, it was difficult to detect a SEC signal. Using 2D TEE, SEC was detected in four patients in the paroxysmal AFib group, and in 47 in long-standing AFib patients, and these differences were statistically significant $(\mathrm{P}<0.001)$ (Table 2).

\section{D TEE echocardiography results}

\section{Diameter and area of LAA orifice in AFib patients}

The LAA (Figure $2 A, B, C, D$, red arrowhead) can be seen in varying sizes during the different phases of the cardiac cycle (the yellow arrow points to the time frame of the cycle). As demonstrated in Figure 2, patients with longstanding AFib showed a different contraction and relaxation LAA morphology during AFib, noting that a coumadin ridge was found in the same patient (Figure 2, asterisk). LAA morphology parameters, including depth, orifice diameter, and orifice area should be strictly assessed in the same cardiac cycle. In order to discover more LAA details and differences between the two AFib classifications, these LAA morphology parameters were further collected and compared by RT-3D TEE. We found that the LAA orifice was markedly enlarged in long-standing patients, compared with paroxysmal AFib group (Figure 3, Table 3). The diameter of the LAA orifice was carefully measured from multiple angles, including the $45^{\circ}$ and $135^{\circ}$ views. The average systolic inner diameter of the LAA opening in the $135^{\circ}$ view were $23.9 \pm 8.4$ and $28.7 \pm 7.6 \mathrm{~mm}$, in the paroxysmal and long-standing groups, respectively $(\mathrm{P}=0.03)$. The average diastolic inner diameter of the LAA opening in the $135^{\circ}$ view were $19.3 \pm 8.9$ and $24.0 \pm 10.1$ $\mathrm{mm}$ in paroxysmal and long-standing groups, respectively $(\mathrm{P}=0.04)$. Meanwhile, there was no difference in terms of the LAA orifice diameter in the $45^{\circ}$ view $(\mathrm{P}=0.05$ in systole, $\mathrm{P}=0.13$ in diastole). Long-standing AFib group patients also had a markedly greater value of LAA area in the systolic and diastolic phases compared with the paroxysmal group $(\mathrm{P}=0.01)$. However, the LAA depth did not differ significantly between them $(30.2 \pm 13.6$ vs. $32.4 \pm 15.8$, respectively, $\mathrm{P}=0.43$ ) (Table 3).

\section{LAA lobes and morphology typing in AFib patients}

We also used RT-3D TEE as a tool to determine the number of LAA lobes, and to identify the relationship 
Table 2 Comparison of 2D TTE/TEE parameters of atrial fibrillation patients in this study

\begin{tabular}{|c|c|c|c|}
\hline Parameters & Paroxysmal AFib (N=110) & Long persistent AFib $(\mathrm{N}=210)$ & $P$ value \\
\hline \multicolumn{4}{|l|}{ 2D TTE } \\
\hline AP LA diameter (mm) & $35.3 \pm 7.2$ & $43.0 \pm 6.8$ & $<0.001$ \\
\hline Systolic LA volume (mL) & $52.6 \pm 27.3$ & $72.8 \pm 21.6$ & $<0.001$ \\
\hline Diastolic LA volume (mL) & $24.5 \pm 12.0$ & $48.3 \pm 15.9$ & $<0.001$ \\
\hline e' septal wave (cm/s) & $6.4 \pm 2.3$ & $6.0 \pm 2.8$ & 0.04 \\
\hline e' lateral wave $(\mathrm{cm} / \mathrm{s})$ & $11.2 \pm 3.6$ & $10.3 \pm 4.1$ & 0.06 \\
\hline$E / e^{\prime}$ & $8.6 \pm 3.5$ & $12.9 \pm 4.2$ & $<0.001$ \\
\hline LV EF\% & $64.4 \pm 10.7$ & $61.8 \pm 11.4$ & 0.34 \\
\hline \multicolumn{4}{|l|}{ 2D TEE } \\
\hline Systolic LAA volume (mL) & $16.9 \pm 7.6$ & $20.4 \pm 11.0$ & 0.03 \\
\hline LAA EF\% & $62.2 \pm 9.5$ & $50.7 \pm 10.3$ & 0.01 \\
\hline Spontaneous echo contrast & $4(3.6 \%)$ & $47(22.4 \%)$ & $<0.001$ \\
\hline
\end{tabular}

All data are presented as number (\%) or mean \pm SD; 2D TTE, two-dimensional transthoracic echocardiography; 2D TEE, two-dimensional transesophageal echocardiography; AP, antero-posterior; LA, left atrium; AFib, atrial fibrillation; LAA, left atrial appendage; LAA EF\%, left atrial appendage ejection fraction.
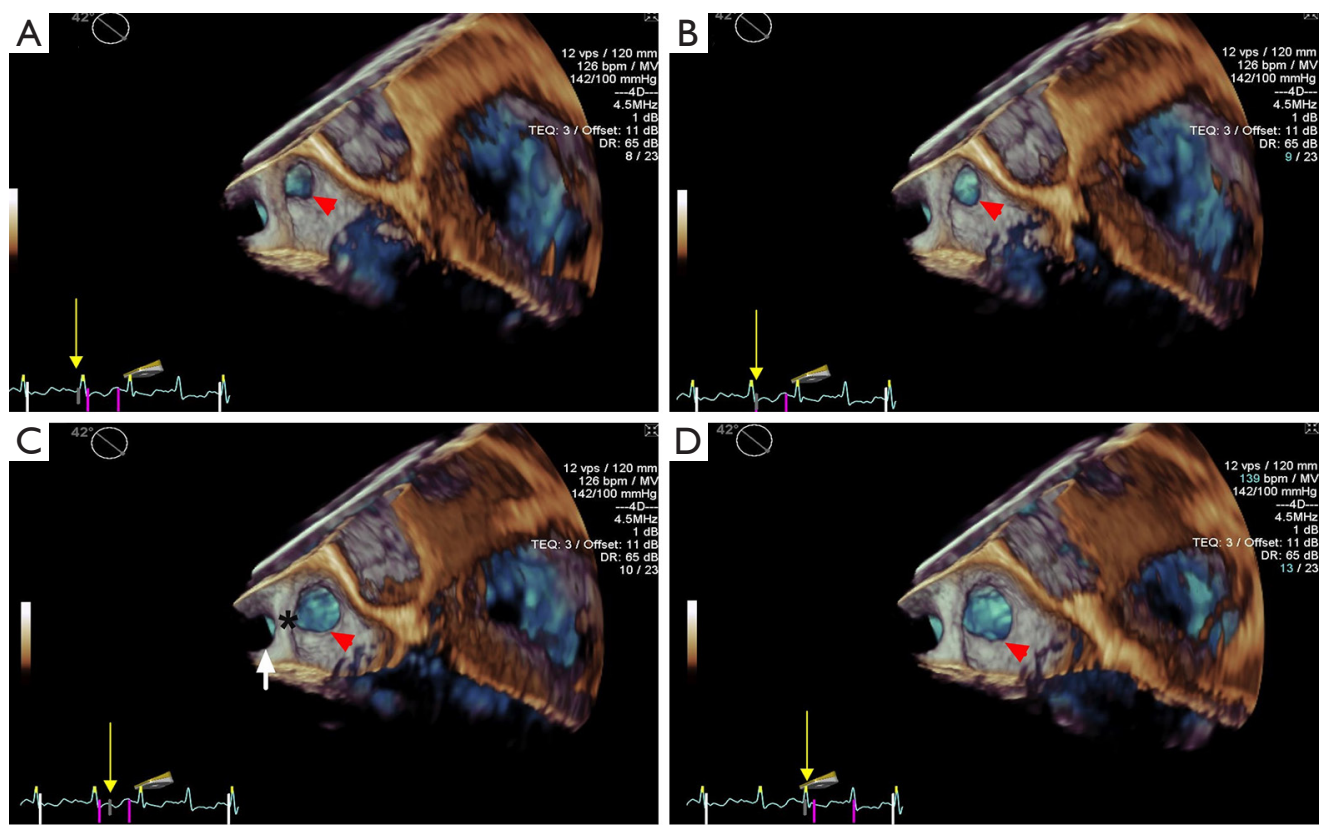

Figure 2 Change in size of the LAA during the cardiac cycle in a patient with AFib. The LAA (red arrowhead) could be seen in varying sizes during the different phases (A,B,C,D) of the cardiac cycle (yellow and thin arrow pointing to time frame of cycle). In addition, a coumadin ridge (asterisk in $\mathrm{C}$ ) and left inferior pulmonary vein (white arrow in $\mathrm{C}$ ) could be clearly seen in this patient. LAA, left atrial appendage; AFib, atrial fibrillation. 

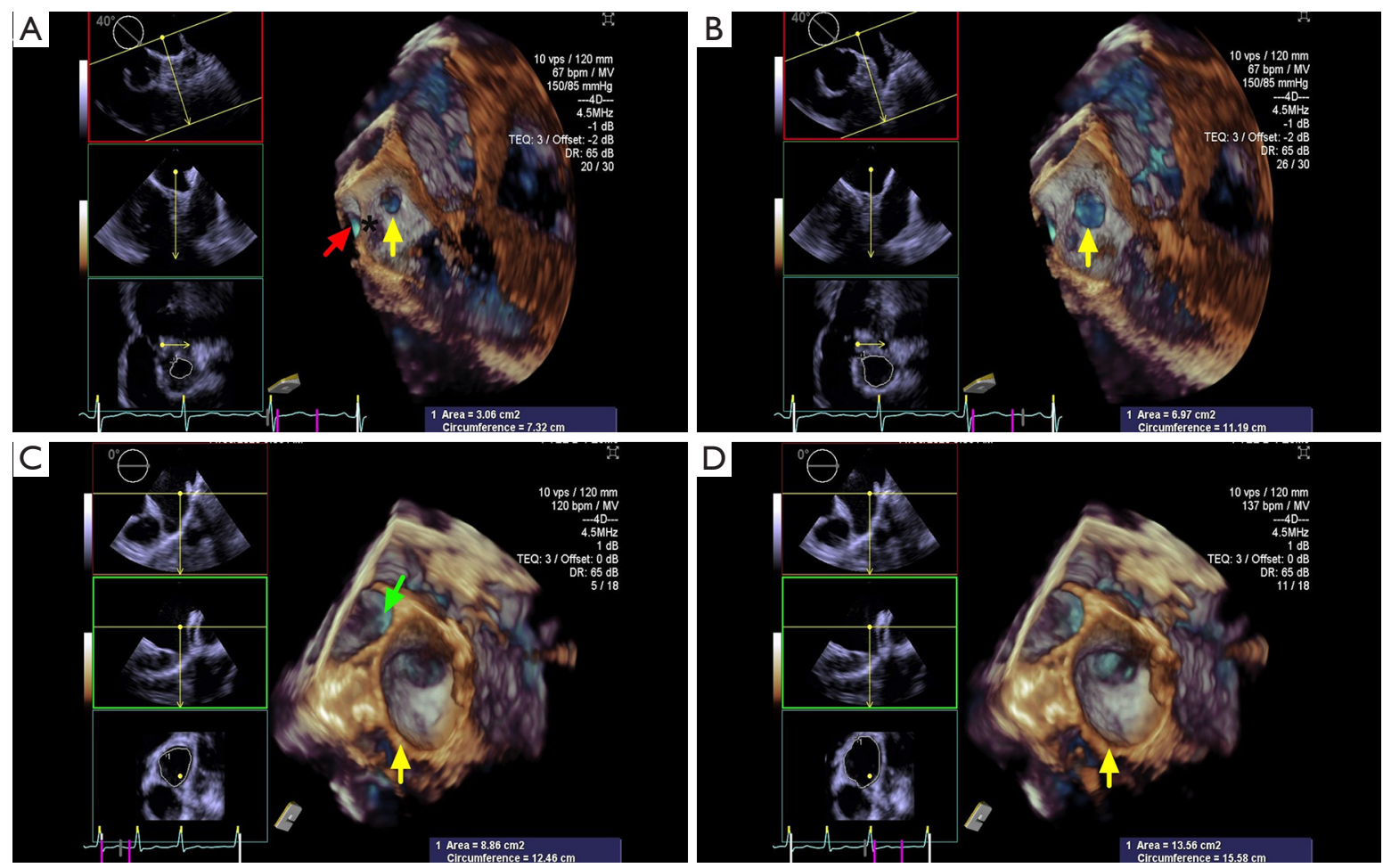

Figure 3 Diameter and area of LAA orifice changes in AFib patients during the cardiac cycle. (Top) A paroxysmal AFib patient. Systole (A) and diastole (B). (Bottom) Another patient with long-lasting AFib. Systole (C) and diastole (D). In the long-lasting AFib patient, the LAA orifice is markedly enlarged; note that the coumadin ridge (asterisk in A), left inferior pulmonary vein (red arrow in A), and left superior pulmonary vein (green arrow in $\mathrm{C}$ ) could be clearly seen in the paroxysmal AFib patient. AFib is associated with structural remodeling of the LAA, which includes dilation of the chamber and reduction in pectinate muscles (not shown). Yellow arrows indicate orifice of LAA in different cardiac cycle. LAA, left atrial appendage; AFib, atrial fibrillation.

between the number of lobes and the AFib types. We found that the majority of LAAs had between two and three lobes, while one lobe accounted for a relatively small proportion in our cohort study (Table 3). Also, we identified a trend of long-standing AFib patients having more LAA lobes compared to paroxysmal group patients, however no statistical difference was found $(\mathrm{P}=0.19)$. In the paroxysmal AFib group, the LAA morphology typing were ordered as follows: cactus type (46\%), cauliflower type (19\%), chicken wing type (20\%), and windsock type (15\%), compared to cactus type (40\%), cauliflower type (32\%), chicken wing type $(16 \%)$, and windsock type (12\%) in the long-standing group (Table 3, Figure 4). The percentage of cauliflower type LAAs in the long-standing AFib group increased significantly $(\mathrm{P}<0.001)$, whereas the proportion of noncauliflower type LAAs decreased slightly, compared with the paroxysmal AFib group $(\mathrm{P} \geq 0.05)$.

\section{SEC, thrombus and relationship with LAA morphological typing in AFib patients}

RT-3D TEE could detect LAA SEC and sludge visually. We defined four grades according to the severity SEC by $3 \mathrm{D}$ TEE. Four $(3.6 \%)$ patients in the paroxysmal AFib group had mild SEC; no severe SEC was detected in these patients. However, there were $28(13.3 \%)$ patients with LAA SEC grades 3-4, and 19 (9.1\%) patients with LAA SEC grades 1-2 in the long-standing AFib group, and the differences were statistically significant $(\mathrm{P}<0.001)$. With regards to LAA thrombus clot, we evaluated the 2D TEE and $3 \mathrm{D}$ TEE in the LAA long axis view, and 3D TEE in the superior view into LAA, in all patients (Figure 5). Most paroxysmal AFib patients had normal LAA morphology and no SEC or thrombi (Figure $5 A, B, C$ ). SEC was definitely more likely to occur in the long-standing AFib patients (Figure 5D,E,F, G,H,I). In addition, the long-standing AFib 
Table 3 Comparison of 3D TEE parameters in different types atrial fibrillation patients

\begin{tabular}{|c|c|c|c|}
\hline Parameters & Paroxysmal AFib $(\mathrm{N}=110)$ & Long persistent AFib (N=210) & $P$ value \\
\hline Systolic LAA Orifice diameter $135^{\circ}(\mathrm{mm})$ & $23.9 \pm 8.4$ & $28.7 \pm 7.6$ & 0.03 \\
\hline Diastolic LAA orifice diameter $135^{\circ}(\mathrm{mm})$ & $19.3 \pm 8.9$ & $24.0 \pm 10.1$ & 0.04 \\
\hline Systolic LAA orifice diameter $45^{\circ}(\mathrm{mm})$ & $17.5 \pm 9.2$ & $20.2 \pm 8.8$ & 0.05 \\
\hline Systolic LAA orifice area $\left(\mathrm{cm}^{2}\right)$ & $4.1 \pm 3.1$ & $7.9 \pm 3.9$ & 0.01 \\
\hline Diastolic LAA orifice area $\left(\mathrm{cm}^{2}\right)$ & $2.7 \pm 1.6$ & $4.2 \pm 2.3$ & 0.01 \\
\hline LAA lobes (No.) & $2.0 \pm 1.3$ & $2.5 \pm 1.8$ & 0.19 \\
\hline \multicolumn{4}{|l|}{ LAA type } \\
\hline Windsock & $16(15 \%)$ & $27(13 \%)$ & 0.56 \\
\hline Cauliflower & $21(19 \%)$ & $74(35 \%)$ & $<0.001$ \\
\hline \multicolumn{4}{|l|}{ LAA SEC } \\
\hline LAA SEC grade 0 & $106(96.4 \%)$ & $163(77.6 \%)$ & $<0.01$ \\
\hline LAA SEC grade $1-2$ & $4(3.6 \%)$ & $19(9.1 \%)$ & $<0.001$ \\
\hline LAA SEC grade $3-4$ & $0(0 \%)$ & $28(13.3 \%)$ & $<0.001$ \\
\hline Thrombus & $2(1.8 \%)$ & 22 (11.5\%) & $<0.001$ \\
\hline
\end{tabular}

All data are presented as number (\%) or mean \pm SD; RT-3D TEE, real-time three-dimensional transesophageal echocardiography; LAA, left atrial appendage; SEC, spontaneous echo contrast; AFib, atrial fibrillation.

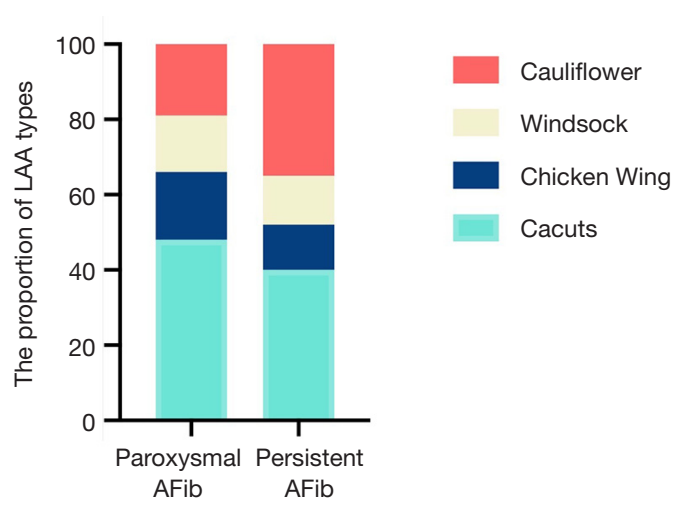

Figure 4 The proportion of LAA types in paroxysmal and longlasting AFib patients. Four LAA types were classification by RT3D TEE, including cauliflower, cactus, windsock, and chicken wing types. LAA, left atrial appendage; AFib, atrial fibrillation. group had a much higher thrombus occurrence rate, and thrombi were found in $22(11.5 \%)$ patients compared with 2 $(1.8 \%)$ in the paroxysmal AFib group $(\mathrm{P}<0.001)$. Moreover, with regard to diagnosis rate, $2 \mathrm{D}$ TEE mistook the acoustic artifact from crest (used for lobulation) as SEC, and even thrombus, in some cases (Figure $5 \mathcal{F}, K, L$ ). The uncertainty of thrombi detection was significantly decreased by $3 \mathrm{D}$ TEE compared with 2D TEE $(\mathrm{P}<0.001)$, and the certainty of thrombi detection by 3D TEE also decreased slightly $(\mathrm{P}=0.06)$. In our study cohort, the rate of thrombi and prior stroke/TIA was not very high [24 (7.5\%) and 29 (9.1\%), respectively in the overall study population]. However, in most cases in our research, thrombi were more likely to occur in cauliflower type LAAs [odds ratio (OR) 2.1, 95\% confidence interval (CI): 1.1-8.5, $\mathrm{P}=0.031]$. 

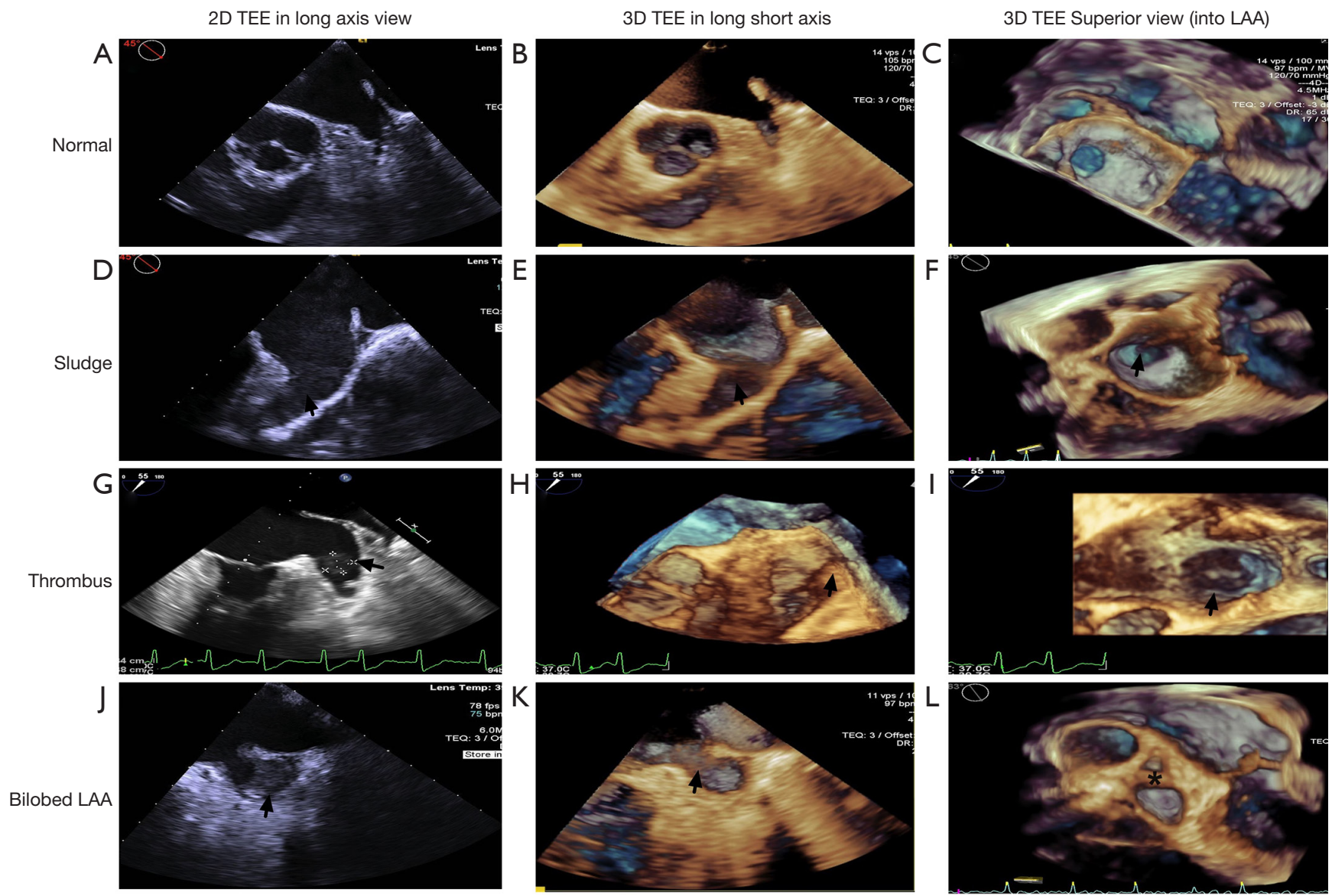

Figure 5 Detection of LAA thrombus clot by 2D/3D TEE in LAA long axis view and 3D TEE superior view into the LAA. A large amount of sludge and SEC could be easily seen by 2D TEE and RT-3D TEE (black arrow in Figure 5D,E,F). We could clearly see the sludge coming out of the LAA orifice (black arrow in Figure 5F). In addition, the thrombus clot was detected by 2D/3D TEE in the LAA long axis view (black arrow in Figure $5 G, H$ ) and by the RT-3D TEE superior view into the LAA; a large thrombus could be detected as well as abundant sludge (black arrow in Figure 5I). In a bilobed LAA, the LAA ridge (black asterisk in Figure 5L) was mistaken for SEC in the 2D TEE LAA long axis view (black arrow in Figure 57,K). With the help of the 3D TEE superior view into the LAA, this acoustic artifact was correctly identified. LAA, left atrial appendage; AFib, atrial fibrillation.

\section{Discussion}

As a reservoir for volume and pressure overload, LAA is normally approximately $10 \%$ of the entire left trial volume, and plays a role in left trial function. Possible multi-plane probes will improve the accuracy of LAA imaging and the diagnosis or exclusion of LAA thrombi (8). Since 2006, several studies have assessed LAA by 3D TTE and 2D TEE (9), and confirmed the value of 3D TEE. Meanwhile, good quality transthoracic images are still the key essential condition. The main aim of our study was to evaluate the value of RT-3D TEE for describing LAA morphology and function, and especially for the detection of LAA thrombi and SEC in paroxysmal and long-standing AFib patients.

Although some studies have demonstrated a high correlation between combined 2D/3D TTE and 2D TEE in the evaluation of LAA thrombi (10), it is important to note that good quality TTE images rely on good penetrability and a perfect acoustic window. In fact, it is too difficult to obtain ideal quality images by TTE to evaluate the morphology and function of LAA, and determine the presence or absence of thrombi. TEE is considered to be superior to TTE due to its markedly higher resolution. The TEE transducer placed within the esophagus is relatively close to the left atrium, which will 
provide more inner cardiac details, including LAA. 2D TEE is usually performed before cardioversion or cardiac radiofrequency ablation to detect the presence of thrombi in AFib/flutter patients who exhibit thromboembolic risk. In our cohort study, all of the enrolled patients underwent TEE examination in a state of superficial larynx anesthesia. We acquired images with the help of skilled cardiac sonographers and cardiologists.

Using 2D TTE and TEE, we observed that some patients in our study had both left atrial chamber and left auricle dilation with AFib. However, some patients had marked dilated left auricle with only slight dilation of the left atrial chamber, while others had dilated left atrial with a normal-sized LAA. We believe that LAA is even more complex than the left atrial main chamber, and the size relationship between them needs to further elucidated. In addition, our study showed that long-standing AFib patients had larger misshaped LAAs (including a dilated orifice and increased LAA volume) compared with paroxysmal AFib patients, which is consistent with previous studies. In our cohort study, we used 2D TEE as the gold standard to assess LAA emptying peak low velocity (LAAeV), which was usually representative of LAA function $(<20 \mathrm{~cm} / \mathrm{sec}$ is considered as LAA dysfunction, while LAAeV $<20 \mathrm{~cm} / \mathrm{sec}$ is associated with the occurrence of SEC and thrombi formation) (11). SEC and thrombi are common in enlarged left atria and LAA. The presence of SEC is the most obvious sign of slow blood flow, and is associated with an increased risk of thromboembolism $(12,13)$. Reduced $\mathrm{LAAeV}$ is regarded as one of the strongest independent predictors of an increased thromboembolic risk (14). Our results showed that $\mathrm{LAAeV}$, supported by $2 \mathrm{D}$ TEE, is highly correlated with the LAA ejection fraction and the incidence of thrombosis, which is consistent with previous studies (1).

Moreover, AFib is usually associated with structural remodeling of the LAA, which includes dilation of the chamber and reduction in pectinate muscles (not shown). However, it is noteworthy that the nature of two dimensions is still the limitation of $2 \mathrm{D}$ TEE. It is impossible to simultaneously obtain multiple views and planes with 2D TEE when measuring opening, depth, and area of LAA, which leads to a biased estimate of LAA size and even to wrong conclusions about the presence or absence of thrombi. In addition, the peripheral auricle structure, such as the coumadin ridge, and slight transverse sinus pericardial fluid accumulation, could produce an acoustic shadowing, which might be confused with real
LAA thrombi. So far, for the evaluation of LAA, few papers have shown that RT-3D TEE is greater than 2D TEE in different classifications of AFib patients. Pourkia et al. (15) described LAA morphologic and functional features in the Iranian population using 3D TEE, but there are few Asian LAA features, especially in Chinese AFib patients. Only a few isolated cases have been reported for thrombus diagnosis; these studies described the accuracy of RT3D TEE in the detection of anatomic variants and LAA thrombi during cardiac surgery or other situations (16). Willens et al. used RT-3D TEE technology to distinguish bilobed LAAs and pectinate muscles from thrombi (17). Yamamoto et al. examined LAA morphology to assess the relationship between the number of lobes and the risk of thrombi formation in AFib patients, and they identified a significant relationship between them (18). Nakajima et al. demonstrated the feasibility of 3D TEE to assess LAA size and geometry in patients with sinus rhythm and AFib (19). 3D TEE was not clinically accepted until user-friendly RT3D TEE was introduced in 2007 (20). The emergence of RT-3D TEE has greatly improved the visualization of the heart structure. This invasive and highly visual technology contributed to the discovery of a substantial amount of heart disease information. In our research, we used it to evaluate LAA morphology, function, and the presence of thrombi. Using 3D TEE, we found that long-standing AFib patients have a greater diameter and area LAA orifice, but the depth changed only slightly. With regards to populations prone to thrombosis, long-standing AFib patients had a higher incidence of both thrombi and SEC than paroxysmal AFib group patients, and exhibited a significantly more severe SEC. These results are consistent with the findings of previous studies (21).

In all AFib patients in our study, the certain thrombus diagnostic rate by $2 \mathrm{D}$ TEE was slightly higher than $3 \mathrm{D}$ TEE, and the uncertain thrombus diagnostic rate by $3 \mathrm{D}$ TEE dramatically decreased compared with 2D TEE. This is because in some particular situations, 2D TEE could not distinguish the pectinate muscles at the bottom of LAA from the small thrombus very well, whereas 3D TEE could easily distinguish them due to the highly-visualized surrounding and internal structure. RT-3D TEE could also clearly identify the surrounding structures, including the coumadin ridge, left upper pulmonary veins, coronary sinus, aortic and mitral valves, etc.

Furthermore, in our study, the variability of LAA lobes in AFib patients was evaluated properly according to previous standards (18). Generally, the lobes pointed toward 
the atrioventricular groove and the basal surface of the left ventricle. This aspect has to be kept in mind during imaging studies, in order to rule out the presence of intracavitary thrombi. Failure to view all of the lobes or incomplete visualization of a lobe may account for the under-diagnosis of LAA thrombosis. We also found that bilobed LAAs accounted for the largest part, followed by trilobed, and then single-lobed (19). However, our understanding of the relationship between of LAA lobes and AFib types remains lacking. We hope to identify the clinical implications of this relationship in the future using $3 \mathrm{D}$ TEE.

With respect to LAA typing, we found that the cactus and cauliflower types accounted for the largest proportion ( $43 \%$ and $30 \%$, respectively) in all patients, while the chicken wing type only accounted for $14 \%$ in our study. However, this finding is not consistent with previous research (22). Di Biase et al. found that the chicken wing type accounted for the largest proportion (48\%), followed by the cactus type (30\%), and then by the windsock (19\%) and cauliflower (3\%) types. Several possible explanations may account for the LAA typing differences. We suggest one reason is that racial differences may play a role in LAA type variation. A second possible reason is that the different detection methods used may have caused the variations. In their study, Di Biase et al. used CT and magnetic resonance imaging (MRI) scans to determine LAA type, whereas our team used several echo methods. More research is needed to reveal this relationship. In addition, the proportion of LAA cauliflower type and the incidence of thrombi dramatically increased in the long-standing AFib group in our study. We suspect that patients with cauliflower LAA morphology have a higher probability of thrombus formation. If confirmed, these results could have a relevant impact on the anticoagulation management of patients with a low/ intermediate risk of stroke/TIA. This may indicate LAA morphology may be a predictor in atrial fibrillation patients who are taken the oral anticoagulant medicine or not. Due to the irregular rhythm of AFib, the $3 \mathrm{D}$ pyramidal fullvolume images were not always available in AFib patients in whom we used 3D Zoom modality to acquire images. It has lower frame rate, but is still a good complementary method that is clinically acceptable. Our results suggested that LAA 3D TEE parameters and images be made available and strongly support clinical results for the assessment of LAA morphology and function.

By virtue of its ability to minimize major bleeding, particularly hemorrhagic stroke, LAAC results in less disability or death than warfarin (23). At present, with the rapid development of cardiac intervention, such as the LAA occlusion technique in AFib patients, it has become especially important to accurately assess the shape and area of the orifice, neck, and depth of LAA prior to the surgery, and to ensure close intraoperative monitoring and postoperative follow-up (24). The most commonly used technique in clinical practice is LAA occlusion via percutaneous catheter under the guidance of fluoroscopy and TEE. However, guided with the fluoroscopy, the complex operation process, the long operating time will not only increase the exposure time of operators, and patients to X-rays, but also the incidence of complications. TEE is a visual way to demonstrate the shape of the LAA, the diameter of the LAA orifice and neck, and the location of the occlusion device. And, 2D and 3D TEE avoid exposure to radiation, shorten the operating time greatly, as well as reduce perioperative complications. RT-3D TEE provides more useful information for a LAA occlusion device than 2D TEE. However, more attention should be paid to the fact that the orifice area will change between the proximal and distal parts of LAA. Thus, intuitively reconstructed 3D TEE images could help us make an appropriate choice with regards to device selection. Moreover, 3D TEE displays the peripheral structure of LAA, such as pulmonary veins and coumadin ridge, which will provide more information during the occlusion process. In addition, 3D TEE offers unique advantages in displaying atrial septum and fossa ovalis compared to other methods, which will help much in guiding puncture before surgery.

\section{Study limitations}

Firstly, 3D full volume images inevitably involve stitched noise due to of the ultimate image composition with different cardiac cycles beat-by-beat. In our study, the usage of the RT-3D TEE Zoom mode (single cardiac cycle image acquisition method) in arrhythmia AFib patients aimed to reduce stitching artifacts, as opposed to using the $3 \mathrm{D}$ full volume mode. However, less higher quantitative images were obtained. Further prospective research is required to validate the use of the $3 \mathrm{D}$ TEE Zoom mode in patients to assess the exact role of RT-3D TEE in evaluating the LAA thrombi. Secondly, the presence or absence of LAA thrombi was not confirmed by surgery or other diagnostic "gold standard" imaging modalities, such as cardiac multidetector CT or MRI. However, an expert panel evaluated the 2D/3D TEE images when we encountered a doubtful diagnosis. Thirdly, this was a retrospective study, and thus 
may not represent real-world clinical situations. We should design prospective studies on a larger scale with a much larger number of atrial fibrillation patients. We still need to do a lot of work to determine the exact role of RT 3DTTE in evaluating the LAA for thrombi in the future. Lastly, we did not enroll permanent AFib patients in our study, and thus our findings may not fully represent AFib.

\section{Conclusions}

A complete analysis of transthoracic M-mode, 2D TEE LAAeV, especially RT-3D TEE is necessary in patients with AFib at risk of embolism. Our study showed that 3D TEE is a safe and real-time option for the evaluation of LAA morphology and function. Moreover, RT-3D TEE is feasible, accurate, and showed an additional diagnostic capability for patients with suspected LAA thrombi. Compared with the paroxysmal AFib group, long-standing AFib patients had greater LAA and SEC, as well as a higher incidence of thrombi. In addition, the cauliflower LAA morphology type was associated with a higher prevalence of SEC and thrombi.

\section{Acknowledgments}

Funding: This work was supported by the National Natural Science Foundation of China (NSFC) grant (No. 81700261).

\section{Footnote}

Reporting Checklist: The authors have completed the STROBE reporting checklist. Available at http://dx.doi. org/10.21037/atm-21-1981

Data Sharing Statement: Available at http://dx.doi. org/10.21037/atm-21-1981

Conflicts of Interest: All authors have completed the ICMJE uniform disclosure form (available at http://dx.doi. org/10.21037/atm-21-1981). The authors have no conflicts of interest to declare.

Ethical Statement: The authors are accountable for all aspects of the work in ensuring that questions related to the accuracy or integrity of any part of the work are appropriately investigated and resolved. This study was performed in accordance with the Declaration of Helsinki (as revised in 2013), and the study protocol was approved by the Sun Yat-sen Memorial Hospital ethics committee. Written informed consent was obtained from all patients for $3 \mathrm{D}$ transesophageal echocardiography.

Open Access Statement: This is an Open Access article distributed in accordance with the Creative Commons Attribution-NonCommercial-NoDerivs 4.0 International License (CC BY-NC-ND 4.0), which permits the noncommercial replication and distribution of the article with the strict proviso that no changes or edits are made and the original work is properly cited (including links to both the formal publication through the relevant DOI and the license). See: https://creativecommons.org/licenses/by-nc-nd/4.0/.

\section{References}

1. Dentamaro I, Vestito D, Michelotto E, et al. Evaluation of left atrial appendage function and thrombi in patients with atrial fibrillation: from transthoracic to real time 3D transesophageal echocardiography. Int J Cardiovasc Imaging 2017;33:491-8.

2. Park HC, Shin J, Ban JE, et al. Left atrial appendage: morphology and function in patients with paroxysmal and persistent atrial fibrillation. Int J Cardiovasc Imaging 2013;29:935-44.

3. Lang RM, Badano LP, Tsang W, et al. EAE/ASE recommendations for image acquisition and display using three-dimensional echocardiography. Eur Heart J Cardiovasc Imaging 2012;13:1-46.

4. Pollick C, Taylor D. Assessment of left atrial appendage function by transesophageal echocardiography. Implications for the development of thrombus. Circulation 1991;84:223-31.

5. Wang Y, Di Biase L, Horton RP, et al. Left atrial appendage studied by computed tomography to help planning for appendage closure device placement. J Cardiovasc Electrophysiol 2010;21:973-82.

6. Beigel R, Wunderlich NC, Ho SY, et al. The left atrial appendage: anatomy, function, and noninvasive evaluation. JACC Cardiovasc Imaging 2014;7:1251-65.

7. Fatkin D, Loupas T, Jacobs N, et al. Quantification of blood echogenicity: evaluation of a semiquantitative method of grading spontaneous echo contrast. Ultrasound Med Biol 1995;21:1191-8.

8. Stöllberger C, Ernst G, Bonner E, et al. Left atrial appendage morphology: comparison of transesophageal images and postmortem casts. Z Kardiol 2003;92:303-8. 
9. Agoston I, Xie T, Tiller FL, et al. Assessment of left atrial appendage by live three-dimensional echocardiography: early experience and comparison with transesophageal echocardiography. Echocardiography 2006;23:127-32.

10. Gorani D, Dilic M, Kulic M, et al. Comparison of two and three dimensional transthoracic versus transesophageal echocardiography in evaluation of anatomy and pathology of left atrial apendage. Med Arch 2013;67:318-21.

11. Vestito D, Desantis D, Colonna P. Three-dimensional Transesophageal Echocardiography Demonstration of Left Atrial Appendage Echocontrast Regression after 6 Months Therapy with Dabigatran and not with Warfarin. J Cardiovasc Echogr 2016;26:52-5.

12. Esposito R, Raia R, De Palma D, et al. The role of echocardiography in the management of the sources of embolism. Future Cardiol 2012;8:101-14.

13. Patel SV, Flaker G. Is early cardioversion for atrial fibrillation safe in patients with spontaneous echocardiographic contrast? Clin Cardiol 2008;31:148-52.

14. Takada T, Yasaka M, Nagatsuka K, et al. Blood flow in the left atrial appendage and embolic stroke in nonvalvular atrial fibrillation. Eur Neurol 2001;46:148-52.

15. Pourkia R, Panahi M, Emkanjoo Z, et al. Morphologic and functional features of left atrial appendage in Iranian population: an echocardiographic study. J Cardiovasc Thorac Res 2019;11:230-6.

16. Matyal R, Mahmood F, Chaudhry H, et al. Left atrial appendage thrombus and real-time 3-dimensional transesophageal echocardiography. J Cardiothorac Vasc Anesth 2010;24:977-9.

Cite this article as: Deng B, Nie R, Qiu Q, Wei Y, Liu Y, Lv H, Zheng S, Wang J. 3D transesophageal echocardiography assists in evaluating the morphology, function, and presence of thrombi of left atrial appendage in patients with atrial fibrillation. Ann Transl Med 2021;9(10):876. doi: 10.21037/ atm-21-1981
17. Willens HJ, Qin JX, Keith K, et al. Diagnosis of a bilobed left atrial appendage and pectinate muscles mimicking thrombi on real-time 3-dimensional transesophageal echocardiography. J Ultrasound Med 2010;29:975-80.

18. Yamamoto M, Seo Y, Kawamatsu N, et al. Complex left atrial appendage morphology and left atrial appendage thrombus formation in patients with atrial fibrillation. Circ Cardiovasc Imaging 2014;7:337-43.

19. Nakajima H, Seo Y, Ishizu T, et al. Analysis of the left atrial appendage by three-dimensional transesophageal echocardiography. Am J Cardiol 2010;106:885-92.

20. Hung J, Lang R, Flachskampf F, et al. 3D echocardiography: a review of the current status and future directions. J Am Soc Echocardiogr 2007;20:213-33.

21. Petersen M, Roehrich A, Balzer J, et al. Left atrial appendage morphology is closely associated with specific echocardiographic flow pattern in patients with atrial fibrillation. Europace 2015;17:539-45.

22. Di Biase L, Santangeli P, Anselmino M, et al. Does the left atrial appendage morphology correlate with the risk of stroke in patients with atrial fibrillation? Results from a multicenter study. J Am Coll Cardiol 2012;60:531-8.

23. Reddy VY, Doshi SK, Kar S, et al. 5-Year Outcomes After Left Atrial Appendage Closure: From the PREVAIL and PROTECT AF Trials. J Am Coll Cardiol 2017;70:2964-75.

24. Vainrib AF, Bamira DG, Saric M. Percutaneous Left Atrial Appendage Closure Devices. Current Cardiovascular Imaging Reports 2017;10:40. 